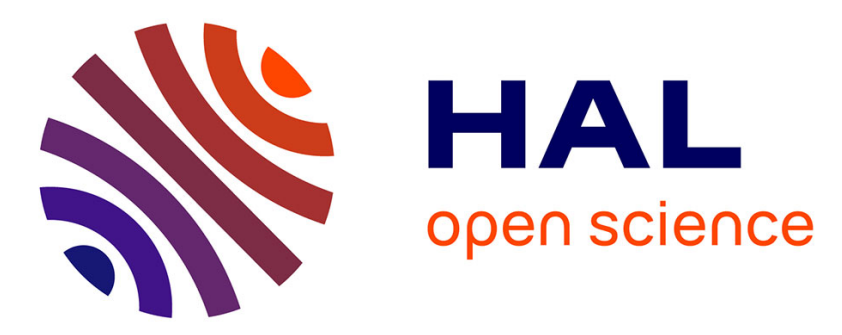

\title{
Indicating the limits of partially automated vehicles with drivers' peripheral vision: an online study
}

Noé Monsaingeon, Yanna Carli, Loïc Caroux, Sabine Langlois, Céline

Lemercier

\section{- To cite this version:}

Noé Monsaingeon, Yanna Carli, Loïc Caroux, Sabine Langlois, Céline Lemercier. Indicating the limits of partially automated vehicles with drivers' peripheral vision: an online study. 12th International Conference on Applied Human Factors and Ergonomics - AHFE 2021, Jul 2021, New York City, NY, United States. pp.78-85. hal-03114194

\section{HAL Id: hal-03114194 https://hal.science/hal-03114194}

Submitted on 12 Feb 2021

HAL is a multi-disciplinary open access archive for the deposit and dissemination of scientific research documents, whether they are published or not. The documents may come from teaching and research institutions in France or abroad, or from public or private research centers.
L'archive ouverte pluridisciplinaire HAL, est destinée au dépôt et à la diffusion de documents scientifiques de niveau recherche, publiés ou non, émanant des établissements d'enseignement et de recherche français ou étrangers, des laboratoires publics ou privés. 


\title{
Indicating the limits of partially automated vehicles with drivers' peripheral vision: an online study
}

\author{
Noé Monsaingeon ${ }^{1,2}$, Yanna Carli ${ }^{1}$, Loïc Caroux ${ }^{2}$, Sabine Langlois ${ }^{1}$, Céline \\ Lemercier $^{2}$ \\ ${ }^{1}$ Renault Technocentre, Guyancourt, France \\ ${ }^{2}$ Laboratoire CLLE, University of Toulouse \& CNRS, Toulouse, France \\ \{noe.monsaingeon; sabine.langlois\}@renault.com \\ yanna.carli@icloud.com \\ \{loic.caroux; celine.lemercier\}@univ-tlse2.fr
}

\begin{abstract}
Automated systems of partially automated vehicles are able to perform the driving task, but can give back the driver all controls in specific conditions. This study aimed to evaluate the effectiveness of an Indicator of Proximity to the Limits of Assistance (IPLA) to anticipate transitions of control. The study was performed online, presenting videos representing situations in which assistance deactivated. A classical interface was compared to an IPLA dedicated to peripheral vision. Participants decided which action to perform. The results revealed that the participants who had the IPLA performed more actions before the system deactivated and expressed greater psychological comfort than the participants with the classic interface. The participants with the IPLA performed less appropriated actions and the IPLA was rated as more cognitively demanding. These results highlight the pertinence of integrating an IPLA dedicated to peripheral vision, but should not encourage the driver to deactivate assistance when not necessary.
\end{abstract}

Keywords: Reliability of automation $\cdot$ Human-machine interface $\cdot$ Online study

\section{Introduction}

In partially automated vehicles, lateral and longitudinal control of the car are delegated to the assistance system. The assistance system of such vehicles is able to perform the driving task in specific conditions, defined by the manufacturer (e.g., lateral acceleration needs to be below a certain threshold). When conditions are no longer met, for example passing through a road with bends with an elevated speed, the support of control ceases, giving back the driver lateral control. When this event occurs, the driver has to be aware that assistance is deactivated in order to take-over control of the car [1]. One goal of interfaces is to avoid as much as possible mode confusions, which are situations in which the driver adopts a behavior that is not adapted to the current state of the assistance [2]. Research on indicators of the reliability of assistance systems showed that these help the driver to anticipate the changes of state of automation [3]. They especially helped to decrease reaction time to take-overs and increase trust in assistance. With partially automated vehicles, Indicators informing on Proximity to the Limits of Assistance (IPLA) revealed to influence the decision to deactivate assistance in several situations that might be confusing for the driver (e.g., road with bends, bad 
weather, unclear road markings) [4]. Kunze et al. [5] tested reliability indicators in peripheral vision for highly automated vehicles, which allowed the driver to reduce take over time without looking off the road. Interfaces presented in peripheral vision, adapted to partially automated vehicles, would help drivers to perform their supervisory task by reacting appropriately in take-over situations, without looking off the road. This study aimed to evaluate the effectiveness of an IPLA, meant to be displayed in peripheral vision, in deciding on appropriate driving behavior in hazardous situations of partially automated vehicles.

An IPLA should dive information through a gradual display, indicating approach from or withdrawal from limits of assistance. This way, the drivers can gauge their responses depending on the emergency of the situation [5]. It has been shown that it is more efficient when an IPLA is state centered, informing on the state and intention of action of assistance [6]. This information needs to be conveyed continuously in order to increase trust in assistance [7]. Such indicators should also not cause cognitive overload with too much additional information [8]. A way to avoid that is to distribute the information on different sensory channels (e.g., auditory, focal vision and peripheral vision) [9]. Interfaces displaying information in peripheral vision allows the driver to free resources of central vision to focus it on the road. With peripheral displays, IPLA should include color hue variations and size variations [5]. The temperature metaphor appears to be efficient to reflect changes in the urgency of a situation, blue reflecting a passive situation and red reflecting a high degree of urgency [10]. Distinctive color steps were reported as efficient for the user to better estimate the urgency of the situation [11]. The changes of color, and therefore urgency, should reflect the state of assistance [7]. Finally, information presented at the top of the cluster should be perceived in peripheral vision [12].

In this study we attempted to answer the following research question: is the IPLA dedicated to peripheral vision, which follows the requirement described above, more adapted than a classical interface to respond to confusing situations? To answer this question, a group using the IPLA interface was compared with a group using a classical interface, referred here as the Reference interface. The following hypotheses were tested during a video-based online study: (1) More decisions to perform actions will be taken before the deactivation of assistance with the IPLA interface; (2) The selected actions will be more adapted to the situation with an IPLA interface; (3) the IPLA interface will induce a better psychological comfort; (4) the IPLA interface will induce a more important cognitive load.

\section{Method}

\section{Participants}

The participants were unpaid French-speaking volunteers, recruited via online social networks and mailing invitations. Half of the participants were coworkers from Renault Technocentre or IRT SystemX. The other half were students from the University of Technology of Compiègne, acquaintances, or family members. The sample was composed of 93 participants, including 30 women. Ages ranged from 18 to more than 65 years, the majority of participants aging from 35 to 49 years. The goal of the study 
was described as the evaluation of a display that aimed to make vehicles' assistance safer. The participants were required to hold a driving licence and to perform the experiment from a computer. The study was explained in the online survey and all participants then completed an informed consent form. Full anonymity was respected.

\section{Videos}

Videos were presented to the participants. The videos depicted four situations from the point of view of a driver of a car in a simulated environment (see Fig. 1). The situations used were reported as situations in which the system can reach its limits in the Renault Clio $52019^{1}$ car user manual. They were selected depending on the conditions, assistance could stop functioning. Four situations were depicted : a road with bends, a traffic jam, a foggy area and an area where road markings were of bad quality. For each of these situations, two videos were presented: one in which the vehicle's assistance deactivated and one in which the vehicle assistance stayed active. This resulted in the presentation of 8 videos. During the road with bends videos, when assistance deactivated, the vehicle went off the road in the bend. During the traffic jam video, the vehicle of the participant braked until it reaches its maximum deceleration, then the emergency braking activated. In the foggy area videos, assistance deactivated due to too much fog density. For the road markings videos, road markings were erased, which resulted in the deactivation of assistance. In the videos, the tested interface was embedded in the cluster and zoomed so that participants could clearly see the information presented whatever the size of their computer displays (see Fig. 1). The videos stopped when the ego vehicle passed the situation or a few seconds after assistance deactivated.

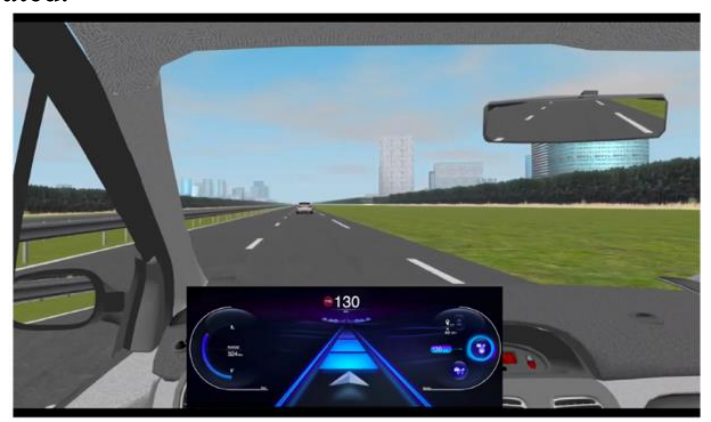

Fig. 1. Screenshot of a presented video during the experimental task for the Reference group. The road scene is represented, as well as the interface.

\section{Interfaces}

Two clusters displays interfaces were compared: an interface equipped with an IPLA and a Reference interface (see Fig. 2). Both interfaces shared mutual characteristics, as follows. The activated level of assistance was displayed at the right of the screen in

\footnotetext{
1 see https://fr.e-guide.renault.com/fra/Clio-5/Assistant-Autoroute-et-Trafic, retrieved on May, 12th, 2020.
} 
blue. When the assistance deactivated, the icon turned grey. The detected road markings were displayed in blue on the screen, as well as horizontal bands representing the distance to the vehicle ahead. Both elements turned grey when assistance deactivated. On the left of the cluster, an area was dedicated to textual messages. The Reference interface displayed only the elements cited previously. The IPLA interface featured additional elements. A glowing halo was displayed at the horizon and represented the proximity to the limits of assistance. It could have three representations : blue and narrow, meaning that assistance is functioning as expected; yellow and medium-sized, meaning that assistance is getting close to its limits but won't disconnect yet; red and large, meaning that assistance is close to its limits of proper functioning and deactivation is very likely to occur. The halo was located in the upper part of the cluster to be perceived in peripheral vision while looking at the road. In addition to the halo, the central zone of the cluster changed depending on the encountered situation. In the road with bends videos, the detected road markings were bent and had the same color as the halo. In the traffic jam videos, the bands representing the distance to the vehicle ahead were the same color as the halo. The foggy area videos, the bands and detected road markings were the same color as the halo. In the bad quality road marking videos, the detected road markings were the same color as the halo. Finally, the textual zone was used to inform the participant of the cause of the approach to the limits of assistance (e.g., erased road markings) and the appropriate action to be taken.

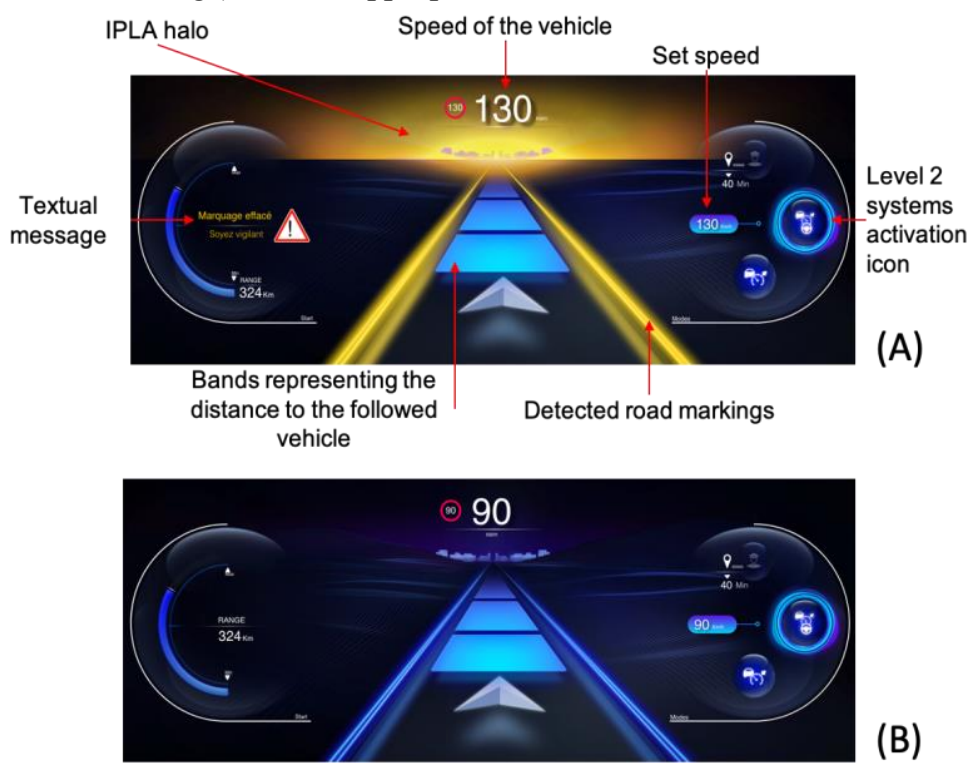

Fig. 2. Screenshots of interfaces presented during the bad quality road markings video. (A) : IPLA; (B) : Reference interface.

\section{Task}

The participants had to watch videos and to project themselves in the presented situations, as if they were driving. They had to choose between the following decisions 
of action when they felt the need to do so, or they could wait for the end of the video: brake, turn the steering wheel, decrease set speed, or deactivate the assistance system. If the participants decided to perform an action, the video stopped.

\section{Procedure}

The participants were randomly assigned to either the IPLA group or the Reference group. They were explained that the vehicle was equipped with an assistance system capable of automatically adapting its speed, lane position, and dealing with bends, but the driver was responsible for monitoring the road, and had to keep their hands on the steering wheel. The assistance system was described as having limited capacities, and that the driver had to take back control if limits were reached. In the IPLA group, participants were instructed that to compensate for the assistance system's limitations, the manufacturer had integrated an indicator. The functioning of the indicator was described. An initial familiarization phase then began and featured two videos in which the vehicle passed next to a highway exit. In one of the two videos, the system deactivated, in the other one, it did not. The subsequent experimental phase began with the 8 videos, presented in a random order. After each video, the participants responded to questions about psychological comfort. After watching all videos, they rated the amount of visual information displayed on the cluster and completed a sociodemographic questionnaire. The survey was implemented on Qualtrics and took less than 15 minutes to be completed $(M=12.86, S D=4.23)$. Full anonymity was respected.

\section{Measures \& Analysis}

Several measures were gathered in this experiment. The first measure was the percentage of participants that decided to perform an action before the system was suspended. A second measure regarded the quality of action in the presented situation. Each action of the participants during the videos were classified as "appropriate" or "inappropriate". The coding was decided before the experiment with two experts in assistance systems. An action was classified as inappropriate if it caused an unnecessary deactivation of the assistance system or if the action was too late to keep the driver safe. An inappropriate action was classified as "too early" if it caused an unnecessary deactivation of the assistance systems. An action was classified as appropriate otherwise. The percentage of appropriate actions was calculated depending on the interface group. A third measure was the psychological comfort experienced after each video, rated by the participants in response to the question "How did you feel during the seconds preceding your action?" on a 6-point scale ranging from 1 (Not good at all) to 6 (Very good). Finally, the cognitive load caused by the interface was rated by the participant with the following question "Of all the trips you took, how would you rate the amount of information displayed on the cluster?" on a 5-point scale ranging from 1 (Too small) to 5 (Too much), asked at the end of the experiment. The IPLA and Reference groups were compared using independent student t-tests. 


\section{Results}

The analysis of the percentage of taken action before deactivation of the assistance revealed that in the road with bends video in which the system suspended, the participants with the IPLA did more actions $(M=75 \% ; S D=44 \%)$ than the participants with the Reference interface $(M=11 \% ; S D=30 \% ; t(91)=8.33 ; p<0.001)$. The analysis on the other videos did not reveal significant differences between the interface groups $(p>.05)$.

The analysis of the quality of the performed actions revealed several significant differences. The participants with the Reference interface did more appropriate actions than the participants with the IPLA during the road with bends video with deactivation of the assistance $(t(91)=-5.2 ; p<0.001)$, without deactivation $(t(91)=-5 ; p<0.001)$, during the traffic jam video without deactivation of assistance $(t(91)=-2.2 ; p=0.03)$ and during the video of bad quality road markings without deactivation of the system $(t(91)=-2.4 ; p=0.01)$. However, the participants with the IPLA did more appropriate actions in the foggy area video with deactivation of assistance $(t(91)=2.4 ; p=0.01$; see Table 1). The analysis of the other videos did not reveal significant differences $(p$ $>$.05). When looking at the number of "too early" inappropriate actions during the road with bends videos with deactivation, a greater number of participants with the IPLA interface did "too early" inappropriate actions $(n=26)$ than the participants with the Reference interface ( $n=0$. For the road with bends video without deactivation, more participants with the IPLA interface did "too early" inappropriate actions $(n=17)$ than the participants with the Reference interface $(n=2)$.

Table 1. Mean percentage of appropriate action (SD) depending on the interface group and video. $* * p<.05 ; * * * p<.001$

\begin{tabular}{llll}
\hline Situation & State of assistance & IPLA group & Reference Group \\
\hline Road with bends & Deactivation & $27 \%(45 \%) * * *$ & $75 \%(43 \%)$ \\
& No deactivation & $50 \%(51 \%)^{* * *}$ & $92 \%(28 \%)$ \\
Traffic jam & Deactivation & $62 \%(49 \%)$ & $63 \%(49 \%)$ \\
\multirow{5}{*}{ Foggy area } & No deactivation & $62 \%(49 \%) * * *$ & $82 \%(39 \%)$ \\
\multirow{3}{*}{ Bad quality road markings area } & Deactivation & $43 \%(50 \%) * *$ & $20 \%(41 \%)$ \\
& No deactivation & $91 \%(29 \%)$ & $98 \%(14 \%)$ \\
& Deactivation & $61 \%(49 \%)$ & $76 \%(43 \%)$ \\
& No deactivation & $84 \%(37 \%) * *$ & $98 \%(14 \%)$ \\
\hline
\end{tabular}

The analysis of psychological comfort revealed a significant difference between interface groups for the road with bends video when the assistance deactivated $(t(89)=$ $3.1 ; p=0.002)$. The participants with the IPLA experienced a better psychological comfort $(M=3.88 ; S D=1.14)$ than the participants with the Reference interface $(M=$ $3.00 ; S D=1.50)$. There was a similar difference for the road with bends video when the assistance did not deactivate $(t(50)=2.2 ; p=0.02)$. The participants with the IPLA experienced a better psychological comfort $(M=4.10 ; S D=1.01)$ than the participants with the Reference interface $(M=3.4 ; S D=0.68)$. The analysis of the other videos did not reveal any significant differences $(p>.05)$. 
The analysis of cognitive load revealed a significant difference between the interface groups. The participants with the Reference interface $(M=3.43 ; S D=0.94)$ rated that their interface was less cluttered with information compared to the participants with the IPLA $(M=4.09 ; S D=0.98 ; t(91)=3.3 ; p=0.001)$.

\section{Discussion}

The participants of the IPLA group did more actions before deactivation of the assistance in the road with bends video. This reflects the fact that the IPLA impacted the decision of the participants to perform an action. Hypothesis (1) was therefore verified, but only for this video. This revealed that the IPLA helped the participants to react before the system deactivated, potentially avoiding confusion. The halo was perceived and impacted the action of the participants, which follows Kunze et al.'s results [5] with highly automated vehicles. However, the participants' actions were not always the most appropriated. Hypothesis (2) was partially verified, because the participants of the Reference group did more actions appropriate to the situation compared to the IPLA group for the road with bends videos with and without deactivation of the assistance, for the traffic jam video with deactivation, and for the bad quality road markings video without deactivation. This could be explained by the fact that the halo used only a three-color gradations. Drivers being cautious, they might rather perform an action that will assure them safety, even if it implies deactivating assistance when IPLA indicated a medium approach to the limits. This is confirmed by the fact that a greater number of participants did "too early" inappropriate actions in the IPLA group, causing unnecessary deactivation of assistance. Following Kunze et al.,'s [6] recommendations, IPLA should be gradual. In our case, the IPLA might have not been gradual enough, presenting alerting information too early relative to the approaching event. However, participants with the IPLA did more appropriate actions during the foggy area video with deactivation of assistance. The conveyed information regarding bad weather may have reassured the participants and helped them to react properly. Regarding psychological comfort, Hypothesis (3) was partially verified. During the seconds preceding the participants' decision of action, participants with the IPLA reported better comfort compared to participants with the Reference interface for the road with bends video, whether the system deactivated or not. Our results follow those of Beller et al. [3], the IPLA made participants feel more psychologically comfortable, increasing acceptability of the assistance. Interestingly, this is the case for videos in which participants did not react appropriately to the situation. Finally, regarding cognitive load, Hypothesis (4) was verified. The participants with the IPLA evaluated the amount of information of the interface as more substantial than the participants with the Reference interface. However, both groups rated their interface as having too much information displayed (at least 3.4 out of 5). The amount of information given by the IPLA seems to be too important, with the interplay of different parts of the cluster. It should be reduced in order not to cause cognitive overload [10]. The main limit of this study was that it was performed online, through videos. The reactions of the participants to interfaces and the size of computer displays are not exactly representative of reality. Simulator experiments would allow to evaluate the efficiency of the IPLA in more ecological situations. 


\section{$5 \quad$ References}

1. Carsten, O., Martens, M.H.: How can humans understand their automated cars? HMI principles, problems and solutions. Cognition, Technology \& Work. 21, 320 (2019). https://doi.org/10.1007/s10111-018-0484-0.

2. Sarter, N.B., Woods, D.D., Billings, C.E.: AUTOMATION SURPRISES. Handbook of human factors and ergonomics. Vol. 2, 1926--1943 (1997).

3. Beller, J., Heesen, M., Vollrath, M.: Improving the Driver-Automation Interaction: An Approach Using Automation Uncertainty. Human Factors. 12 (2013).

4. Monsaingeon, N., Caroux, L., Langlois, S., Hurgobin, Y., Lemercier, C.: Driver compliance with automation reliability information regarding hazardous environmental circumstances. Le travail humain. Vol. 83, 343-360 (2020).

5. Kunze, A., Summerskill, S.J., Marshall, R., Filtness, A.J.: Conveying Uncertainties Using Peripheral Awareness Displays in the Context of Automated Driving. In: Proceedings of the 11th International Conference on Automotive User Interfaces and Interactive Vehicular Applications - AutomotiveUI '19. pp. 329-341. ACM Press, Utrecht, Netherlands (2019). https://doi.org/10.1145/3342197.3344537.

6. Noah, B.E., Gable, T.M., Chen, S.-Y., Singh, S., Walker, B.N.: Development and Preliminary Evaluation of Reliability Displays for Automated Lane Keeping. In: Proceedings of the 9th International Conference on Automotive User Interfaces and Interactive Vehicular Applications - AutomotiveUI '17. pp. 202-208. ACM Press, Oldenburg, Germany (2017). https://doi.org/10.1145/3122986.3123007.

7. Seppelt, B.D., Lee, J.D.: Making adaptive cruise control (ACC) limits visible. International Journal of Human-Computer Studies. 65, 192-205 (2007). https://doi.org/10.1016/j.ijhcs.2006.10.001.

8. Mankoff, J., Dey, A.K., Hsieh, G., Kientz, J., Lederer, S., Ames, M.: Heuristic evaluation of ambient displays. In: Proceedings of the SIGCHI Conference on Human Factors in Computing Systems. pp. 169-176. Association for Computing Machinery, New York, NY, USA (2003). https://doi.org/10.1145/642611.642642.

9. Politis, I., Brewster, S.A., and Pollick F. Evaluating multimodal driver displays under varying situational urgency. In Proceedings of the SIGCHI Conference on Human Factors in Computing Systems - CHI '14. pp. 4067-4076. Association for Computing Machinery, New York, NY, USA (2014). DOI:https://doi.org/10.1145/2556288.255698.

10. Davis, K., Owusu, E.B., Marcenaro, L., Feijs, L., Regazzoni, C., Hu, J.: Effects of Ambient Lighting Displays on Peripheral Activity Awareness. IEEE Access. 5, 9318-9335 (2017). https://doi.org/10.1109/ACCESS.2017.2703866.

11. Faltaous, S., Baumann, M., Schneegass, S., Chuang, L.L.: Design Guidelines for Reliability Communication in Autonomous Vehicles. In: Proceedings of the 10th International Conference on Automotive User Interfaces and Interactive Vehicular Applications - AutomotiveUI '18. pp. 258-267. ACM Press, Toronto, ON, Canada (2018). https://doi.org/10.1145/3239060.3239072.

12. Terken, J., Riener, A., Schroeter, R., Osswald, S.: Adjunct Proceedings of the 5th International Conference on Automotive User Interfaces and Interactive Vehicular Applications. (2013). 